\title{
Letter
}

\section{Quantum-gravity fluctuations and the black-hole temperature}

\author{
Shahar Hod ${ }^{1,2, \mathrm{a}}$ \\ ${ }^{1}$ The Ruppin Academic Center, 40250 Emeq Hefer, Israel \\ 2 The Hadassah Institute, 91010 Jerusalem, Israel
}

Received: 3 January 2015 / Accepted: 18 May 2015 / Published online: 29 May 2015

(C) The Author(s) 2015. This article is published with open access at Springerlink.com

\begin{abstract}
Bekenstein has put forward the idea that, in a quantum theory of gravity, a black hole should have a discrete energy spectrum with concomitant discrete line emission. The quantized black-hole radiation spectrum is expected to be very different from Hawking's semi-classical prediction of a thermal black-hole radiation spectrum. One naturally wonders: Is it possible to reconcile the discrete quantum spectrum suggested by Bekenstein with the continuous semi-classical spectrum suggested by Hawking? In order to address this fundamental question, in this essay we shall consider the zero-point quantum-gravity fluctuations of the black-hole spacetime. In a quantum theory of gravity, these spacetime fluctuations are closely related to the characteristic gravitational resonances of the corresponding black-hole spacetime. Assuming that the energy of the black-hole radiation stems from these zero-point quantum-gravity fluctuations of the black-hole spacetime, we derive the effective temperature of the quantized black-hole radiation spectrum. Remarkably, it is shown that this characteristic temperature of the discrete (quantized) black-hole radiation agrees with the well-known Hawking temperature of the continuous (semiclassical) black-hole spectrum.

One of the most remarkable theoretical predictions of modern physics is Hawking's celebrated result that black holes are not completely black [1]. According to Hawking's semi-classical analysis, a black hole is quantum mechanically unstable-it emits continuous thermal radiation whose characteristic temperature is given by
\end{abstract}

$T_{\mathrm{H}}=\frac{\hbar}{8 \pi M}$.

Here $M$ is the mass of the Schwarzschild black hole. (We use gravitational units in which $G=c=1$.)

It should be stressed, however, that Hawking's derivation of the continuous black-hole radiation spectrum is restricted to the semi-classical regime: the matter fields are treated quantum mechanically but the spacetime (and, in particular,

\footnotetext{
a e-mail: shaharhod@gmail.com
}

the black hole itself) are treated classically. One therefore expects to find important new features in the character of the black-hole radiation spectrum once quantum properties of the black hole itself are properly taken into account. ${ }^{1}$ It is therefore appropriate to regard the Hawking temperature (1) as the semi-classical (SC) temperature of the continuous black-hole radiation:

$T_{\mathrm{SC}} \equiv T_{\mathrm{H}}=\frac{\hbar}{8 \pi M}$.

The quantization of black holes was first proposed in the seminal work of Bekenstein [2,3]. The original quantization procedure was based on the physical observation that the surface area of a black hole behaves as a classical adiabatic invariant [2,3]. In the spirit of the Ehrenfest principle [4], any classical adiabatic invariant corresponds to a quantum entity with a discrete spectrum, Bekenstein suggested that the horizon area $A$ of a quantum black hole should have a discrete spectrum of the form

$A_{n}=\gamma \hbar \cdot n ; \quad n=1,2,3, \ldots$

Here $\gamma$ is an unknown "fudge" factor which was introduced in $[2,3]$.

In order to determine the value of the coefficient $\gamma$, Mukhanov and Bekenstein [5,6] have suggested, in the spirit of the Boltzmann-Einstein formula in statistical physics [4], to relate $g_{n} \equiv \exp \left[S_{\mathrm{BH}}(n)\right]$ to the number of blackhole micro-states that correspond to a particular external black-hole macro-state. Here $S_{\mathrm{BH}}$ is the black-hole entropy, which is related to its surface area $A$ by the thermodynamicgeometric relation $[1,2]$

$S_{\mathrm{BH}}=\frac{A}{4 \hbar}$.

\footnotetext{
1 This state of affairs is reminiscent of atomic spectroscopy: according to the classical laws of electrodynamics an atom should have a continuous emission spectrum, whereas quantum mechanics dictates a discrete line emission from the atom.
} 
The statistical degeneracy [see Eqs. (3) and (4)]

$g_{n} \equiv \exp \left[S_{\mathrm{BH}}(n)\right]=\exp \left(\frac{1}{4} \gamma \cdot n\right)$

of the $n$th black-hole area level has to be an integer for every integer $n$. This physical requirement dictates the relation [5, 6]

$\gamma=4 \ln k$

for the fudge factor $\gamma$, where the unknown constant $k$ should be an integer.

Determining the specific value of the integer $k$ requires additional physical input. This physical information emerges by applying the Bohr correspondence principle [4] to the discrete resonance spectrum of the black hole [7]. According to the Bohr correspondence principle, transition frequencies at large quantum numbers should equal classical oscillation frequencies. Namely, the asymptotic energy difference $M_{n+1}-M_{n}$ between the $(n+1)$ th and the $n$th black-hole quantum levels should be given by the characteristic classical oscillation frequency of the black hole:

$M_{n+1}-M_{n}=\hbar \omega_{\mathrm{BH}}$.

It is well known that a Schwarzschild black hole is characterized by a discrete spectrum of gravitational resonances $[8-10]$ with the fundamental asymptotic frequency $[7,11]$

$M \omega_{\mathrm{R}}=\frac{\ln 3}{8 \pi}$.

The emission of a gravitational quantum from the black hole results in a change $\Delta M=\hbar \omega_{R}$ [see Eq. (7)] in the blackhole mass. Using the first-law of black-hole thermodynamics, $\Delta A=32 \pi M \Delta M,^{2}$ one finds the fundamental change $\Delta A=4 \ln 3 \cdot \hbar$ in the Schwarzschild black-hole surface area. Taking cognizance of Eqs. (3) and (8), one finally obtains the quantized area spectrum:

$A_{n}=4 \hbar \ln 3 \cdot n ; \quad n=1,2,3, \ldots$

It is worth emphasizing again that the black-hole area spectrum (9) is consistent both with the area-entropy thermodynamic relation (4) for black holes, with the BoltzmannEinstein formula (5) in statistical physics, and with the Bohr correspondence principle (7) [7].

One therefore concludes that, in a quantum theory of gravity, a Schwarzschild black hole has a discrete energy (mass) spectrum of the form: ${ }^{3}$

$M_{n}=\sqrt{\frac{\hbar \ln 3}{4 \pi}} \cdot n^{1 / 2} ; \quad n=1,2,3, \ldots$,

\footnotetext{
${ }^{2}$ Here we have used the relation $A=16 \pi M^{2}$ for the Schwarzschild black hole.

${ }^{3}$ See footnote 2 .
}

with concomitant discrete line emission [5-7]. In particular, the radiation emitted by the quantized black hole consists of gravitational quanta whose frequencies are integer multiples of the fundamental black-hole frequency

$\omega_{0} \equiv\left(M_{n+1}-M_{n}\right) / \hbar=\frac{\ln 3}{8 \pi M}$.

The quantized (discrete) black-hole radiation spectrum is obviously different from Hawking's semi-classical prediction of a thermal (continuous) spectrum.

One naturally wonders: Is it possible to reconcile the discrete quantum spectrum predicted by Bekenstein with the continuous semi-classical spectrum predicted by Hawking? In order to address this fundamental question, we first point out that, in a quantum theory of gravity, the black-hole spacetime is expected to possess a set of zero-point quantumgravity fluctuations. It has been suggested $[12,13]$ that these zero-point fluctuations of the black-hole spacetime (and, in particular, the quantum-gravity fluctuations of the black-hole horizon) may enable quanta to tunnel out of the black hole.

We shall now conjecture that these black-hole spacetime fluctuations are characterized by the fundamental resonance frequency $\omega_{0}$ [see Eq. (11)] of the black-hole spacetime. In particular, we propose a physical picture in which the quantum-gravity fluctuations of the black-hole spacetime may enable quanta with the appropriate frequencies (the ones which are in resonance with the fluctuating horizon: $\left.\omega_{0}, 2 \omega_{0}, 3 \omega_{0}, \ldots\right)$ to tunnel out of the quantum black hole.

According to this physical picture, the energy of the blackhole radiation stems from these zero-point quantum-gravity fluctuations of the black-hole spacetime. The characteristic quantum temperature, $T_{\mathrm{Q}}$, of the discrete black-hole radiation spectrum may be defined by equating the mean thermal energy of the radiating fields (including their zero-point quantum energy) with the corresponding energy of an emitted quantum with the characteristic black-hole resonance frequency $\omega_{0}$. Namely,

$\frac{1}{2} \hbar \omega_{0}+\frac{\hbar \omega_{0}}{\mathrm{e}^{\hbar \omega_{0} / T_{\mathrm{Q}}}-1}=\hbar \omega_{0}$.

Substituting the fundamental black-hole resonance $\omega_{0}=$ $\ln 3 / 8 \pi M$ [see Eq. (11)] into Eq. (12), one finds that the characteristic temperature of the quantized $(\mathrm{Q})$ black-hole radiation spectrum is given by

$T_{\mathrm{Q}}=\frac{\hbar}{8 \pi M}$.

Remarkably, we find here that the characteristic temperature (13) of the discrete (quantized) black-hole radiation spectrum exactly matches the semi-classical temperature (2) of the continuous black-hole radiation:

$T_{\mathrm{Q}}=T_{\mathrm{SC}}$ 
Summary One of the most important theoretical predictions of modern physics is Hawking's semi-classical ${ }^{4}$ result that black holes are not completely black [1]. In particular, according to Hawking's analysis, a Schwarzschild black hole is expected to emit continuous thermal radiation whose characteristic semi-classical temperature is given by Eq. (2). However, Bekenstein [2,3] has put forward the idea that, in a quantum theory of gravity, ${ }^{5}$ a quantum black hole should have a discrete mass spectrum. As a consequence, a quantum black hole is expected to be characterized by a discrete line emission.

In the present essay we have proposed a physical mechanism which relates in a natural way the two seemingly different spectra: the discrete black-hole quantum spectrum as predicted by Bekenstein and the semi-classical continuous spectrum as predicted by Hawking. The proposed model is based on the fact that, in a quantum theory of gravity, the black-hole spacetime is expected to possess a set of zeropoint quantum-gravity fluctuations which are characterized by the fundamental black-hole resonance frequency (11). These quantum-gravity fluctuations of the black-hole horizon may enable quanta with the appropriate frequencies (the frequencies $\omega_{0}, 2 \omega_{0}, 3 \omega_{0}, \ldots$ which are in resonance with the fluctuating horizon) to tunnel out of the quantum black hole.

The resulting quantum-gravity black-hole radiation spectrum is characterized by a discrete line emission as predicted by Bekenstein. Remarkably, we have shown here that this quantized (discrete) black-hole radiation spectrum is characterized by an effective quantum temperature $T_{\mathrm{Q}}$ [see Eq. (13)] which agrees with the well-known semi-classical Hawking temperature $T_{\mathrm{H}}$ [see Eq. (2)] of the continuous black-hole radiation spectrum.

Acknowledgments This research is supported by the Carmel Science Foundation. I thank Yael Oren, Arbel M. Ongo, Ayelet B. Lata, and Alona B. Tea for stimulating discussions.

Open Access This article is distributed under the terms of the Creative Commons Attribution 4.0 International License (http://creativecomm ons.org/licenses/by/4.0/), which permits unrestricted use, distribution, and reproduction in any medium, provided you give appropriate credit to the original author(s) and the source, provide a link to the Creative Commons license, and indicate if changes were made. Funded by SCOAP ${ }^{3}$.

\section{References}

1. S.W. Hawking, Commun. Math. Phys. 43, 199 (1975)

2. J.D. Bekenstein, Phys. Rev. D 7, 2333 (1973)

3. J.D. Bekenstein, Lett. Nuovo Cimento 11, 467 (1974)

4. M. Born, Atomic Physics (Blackie, London, 1969)

5. V. Mukhanov, JETP Lett. 44, 63 (1986)

6. J.D. Bekenstein, V.F. Mukhanov, Phys. Lett. B 360, 7 (1995)

7. S. Hod, Phys. Rev. Lett. 81, 4293 (1998)

8. H.P. Nollert, Class. Quantum Gravity 16, R159 (1999)

9. E.W. Leaver, Proc. R. Soc. A 402, 285 (1985)

10. H.P. Nollert, Phys. Rev. D 47, 5253 (1993)

11. See S. Hod, Class. Quantum Gravity 23, L23 (2006) [for the physical motivation of focusing on the asymptotic black-hole resonances]

12. J.W. York, Phys. Rev. D 28, 2929 (1983)

13. J.B. Hartle, S.W. Hawking, Phys. Rev. D 13, 2188 (1975)

\footnotetext{
${ }^{4}$ It is worth emphasizing again that, in Hawking's original analysis [1], the matter fields are treated quantum mechanically but the black hole itself is treated as a classical entity. Thus, Hawking's analysis is restricted to the semi-classical regime.

5 In a quantum theory of gravity, the spacetime itself (and, in particular, the black-hole energy spectrum) should be treated as a quantum entity.
} 